\title{
CrystEngComm
}

PAPER
View Article Online

View Journal I View Issue

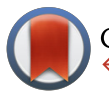

CrossMark \& click for updates

Cite this: CrystEngComm, 2016, 18 , 3211

Received 22nd February 2016, Accepted 1st April 2016

DOI: $10.1039 / c 6 c e 00419 a$

www.rsc.org/crystengcomm

\section{Pressure-induced preference for solvation of 5,6-dimethylbenzimidazole $\uparrow$}

\author{
Witold Zielinski and Andrzej Katrusiak*
}

5,6-Dimethylbenzimidazole $\left(\mathrm{C}_{9} \mathrm{H}_{9} \mathrm{~N}_{2}\right)$ crystallizes exclusively in the unsolvated form from an aqueous solution up to $0.25 \mathrm{GPa}$ and from methanol, ethanol or their mixed solutions up to $0.6 \mathrm{GPa}$. At still higher pressures, solvated crystals with water, methanol and ethanol are formed depending on the solvent used for recrystallization. The $\mathrm{NH} \cdots \mathrm{N}$ bonds of the neat crystals are replaced by $\mathrm{NH} \cdots \mathrm{OH} \cdots \mathrm{N}$ bonds in the solvates. This preference for high-pressure solvation has been rationalized in terms of changed hydrogen bonds as well as volume balance between neat and solvated compounds. The methanol solvate $\mathrm{C}_{9} \mathrm{H}_{9} \mathrm{~N}_{2} \cdot \mathrm{MeOH}$ undergoes a pressure-induced phase transition at $1.40 \mathrm{GPa}$, lowering its symmetry from monoclinic space group $P 2_{1} / C$ (phase $\alpha$ ) to triclinic space group $P \overline{1}$ (high-pressure phase $\beta$ ) and ordering the $\mathrm{H}$-atoms in hydrogen bonds $\mathrm{NH} \cdots \mathrm{OH} \cdots \mathrm{N}$, disordered in phase $\alpha$.

\section{Introduction}

Most organic compounds preferentially form either unsolvated or solvated crystals under normal conditions. There are also substances forming solvates ${ }^{1-6}$ depending on concentration and temperature. ${ }^{7}$ The formation of solvates is often desired in the agriculture and pharmaceutical industry, ${ }^{8}$ because the solvation efficiently modifies such properties as solubility, melting and stability, most important for practical applications. ${ }^{9-11}$ There are also examples of the effect of pressure on the solvation preferences. ${ }^{12}$ Presently we have investigated the effect of pressure for the crystallization of 5,6-dimethylbenzimidazole (dMBzIm). The obtained solvates are most intriguing when compared to the crystallization preferences of analogous benzimidazole (BzIm), ${ }^{13}$ and 2-methylbenzimidazole (MBzIm), ${ }^{14}$ none of which formed any solvates neither at ambient or high pressure. The compression of neat crystals as well as high-pressure recrystallization of BzIm and of MBzIm resulted in the same phases. Recently, we reported the pressure-induced hydration of dMBzIm. ${ }^{15}$ The formation of hemihydrate dMBzIm $\cdot 1 / 2 \mathrm{H}_{2} \mathrm{O}$ above 0.25 GPa is counterintuitive because the molecular volume $(\mathrm{V} / \mathrm{Z})$ of the hemihydrate is significantly smaller than that of neat dMBzIm. Moreover, the hemihydrate crystals recovered to ambient pressure have not changed in open vials for years. Presently we have extended the study on dMBzIm to its highpressure crystallization of methanol and ethanol solutions.

Faculty of Chemistry, Adam Mickiewicz University, Umultowska 89b, 61-614 Poznań, Poland. E-mail: katran@amu.edu.pl

$\dagger$ Electronic supplementary information (ESI) available. CCDC 1451562-1451571. For ESI and crystallographic data in CIF or other electronic format see DOI: 10.1039/c6ce00419a
We found that the crystallization of dMBzIm can be controlled by the pressure, favoring the methanol (dMBzIm.MeOH) and ethanol (dMBzIm·EtOH) solvates. Fig. 1 shows the series of benzimidazole derivatives recrystallized at high pressure, and the obtained dMBzIm solvates. It was our intention to explain this different preferential high-pressure crystallization of BzIm, MBzIm and dMBzIm as well as the solvation of this latter compound.

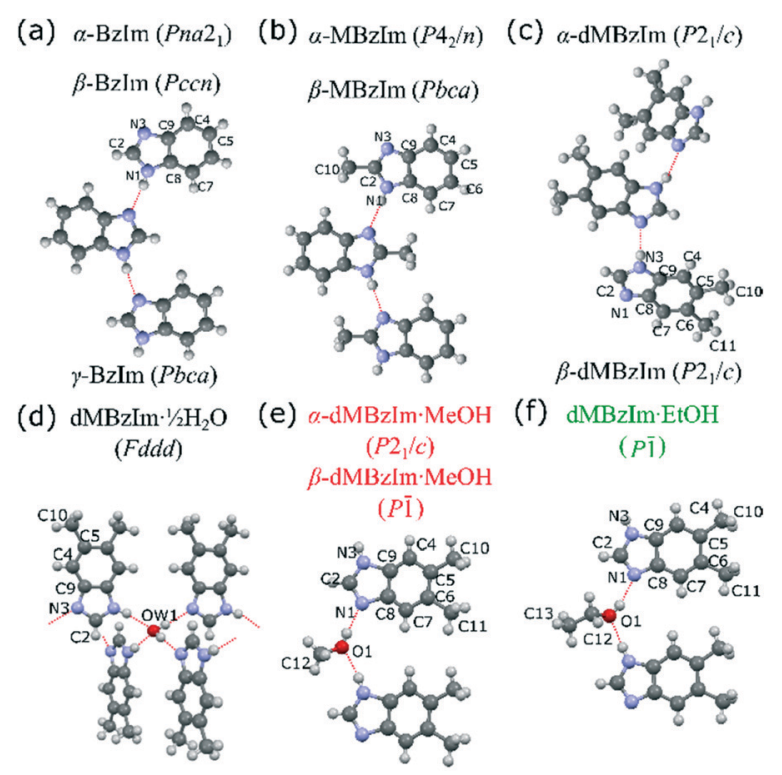

Fig. $1 \mathrm{H}$-Bonded aggregates of benzimidazole, BzIm (a) and its derivatives, 2-methylbenzimidazole, MBzIm (b), and 5,6-dimethylbenzimidazole, dMBzIm (c), as well as the solvates of dMBzIm: hemihydrate dMBzIM.1/2 $\mathrm{H}_{2} \mathrm{O}$ (d), methanol solvate dMBzIm.MeOH (e) and ethanol solvate dMBzIm.EtOH (f). This drawing also lists the high-pressure phases (labeled $\beta$ and $\gamma$ ) of the neat compounds $(\mathrm{a}-\mathrm{c})$ and the methanol solvate (e). 


\section{Results and discussion}

The dMeBzIm crystallization performed above 0.6 GPa from methanol and ethanol solutions resulted in solvates. The crystallization of methanol and of methanol: ethanol:water mixture (16:3:1 vol) solutions yields methanol solvate crystals in a monoclinic system, space group $P 2_{1} / c$. The pressure preference for the solvate formation could be determined visually by recognizing the characteristic elongated plate morphology of neat dMBzIm and rhomboid plates of its solvates (Fig. 6). Likewise, a pressure of $0.6 \mathrm{GPa}$ was required to obtain solvate dMBzIm.EtOH from an ethanol solution. The X-ray diffraction measurements revealed the apparent differences in the lattice dimensions (Table 1 and Fig. 2), the symmetry and the molecular association between neat dMBzIm and its solvates.

The anomalous compression of $\mathrm{dMBzIm} \cdot \mathrm{MeOH}$ indicates its phase transition at $1.40 \mathrm{GPa}$ : the monoclinic phase below $1.40 \mathrm{GPa}$ has been denoted with the Greek letter $\alpha$ and the high-pressure triclinic phase with the letter $\beta$. The phase transition at $1.40 \mathrm{GPa}$ occurred upon increasing pressure, but the crystal cracked and crystallization had to be repeated above $1.40 \mathrm{GPa}$ in order to obtain good quality single crystals for X-ray diffraction studies. The unit-cell dimensions of phases $\alpha$ and $\beta$ are similar, apart from the triclinic strain in phase $\beta$ and the unit-cell volume and contents of phase $\beta$ being half those of phase $\alpha$; parameter $c$ of the triclinic unit cell is halved in the triclinic $\beta$ phase (see Fig. 2 and 3).

In the solvated crystals, the dMBzIm and solvent molecules are $\mathrm{NH}^{\cdots} \cdot \mathrm{OH} \cdots \mathrm{N}$ bonded (Fig. 3 ). In dMBzIm.MeOH the chains run along axis $[y]$ and this direction of the crystal is the least compressed. A similar interval of $\mathrm{H}$-bonded chains parallel to axis $[x]$ in dMBzIm.EtOH is reflected in the similar unit-cell parameter $a$ and its small compressibility. The unitcell of phase $\beta$ has been chosen as the halved unit-cell of phase $\alpha$ (stable between 0.1 MPa and 0.6 GPa) along $[z]$. This choice of unit-cell in phase $\beta$ is not the conventional unit cell, but it allows a convenient comparison of these related structures.

Table 1 Selected crystal data for the high-pressure structures of dMBzlm and the methanol and ethanol solvates, all at $296 \mathrm{~K}$. Low- and highpressure polymorphs of solvate $\mathrm{dMBz} / \mathrm{m} \cdot \mathrm{MeOH}$ are labeled with Greek letters $\alpha$ and $\beta$, respectively

\begin{tabular}{lllll}
\hline & dMBzIm & $\begin{array}{l}\alpha \text {-dMBzIm } \\
\text { MeOH }\end{array}$ & $\begin{array}{l}\beta \text {-dMBzIm } \\
\text { MeOH }\end{array}$ & $\begin{array}{l}\text { dMBzIm } \\
\text { EtOH }\end{array}$ \\
\hline$P(\mathrm{GPa})$ & $0.33(2)$ & $0.92(2)$ & $1.53(2)$ & $1.50(2)$ \\
Space group & $P 2_{1} / c$ & $P 2_{1} / c$ & $P \overline{1}$ & $P \overline{1}$ \\
$a(\AA)$ & $6.538(3)$ & $9.977(7)$ & $10.111(5)$ & $7.0048(7)$ \\
$b(\AA)$ & $26.99(14)$ & $7.1746(3)$ & $7.1012(7)$ & $8.453(3)$ \\
$c(\AA)$ & $13.930(5)$ & $12.7576(6)$ & $6.6980(9)$ & $9.3834(12)$ \\
$\alpha\left(^{\circ}\right)$ & 90 & 90 & $114.719(11)$ & $66.49(2)$ \\
$\beta\left({ }^{\circ}\right)$ & $102.68(4)$ & $93.545(13)$ & $94.60(2)$ & $81.840(9)$ \\
$\gamma\left({ }^{\circ}\right)$ & 90 & 90 & $90.206(18)$ & $71.705(18)$ \\
Volume $\left(\AA^{3}\right)$ & $2398(13)$ & $911.4(6)$ & $435.1(2)$ & $483.6(2)$ \\
$Z / Z^{\prime}$ & $12 / 3$ & $4 / 1$ & $2 / 1$ & $2 / 1$ \\
$D_{\mathrm{x}}\left(\mathrm{g} \mathrm{cm}{ }^{-3}\right)$ & 1.212 & 1.299 & 1.36 & 1.32 \\
$R_{1} / \mathrm{wR}_{2}$ & $0.086 / 0.198$ & $0.050 / 0.129$ & $0.083 / 0.181$ & $0.057 / 0.141$
\end{tabular}

a)

$\mathrm{dMBzIm} \cdot \mathrm{MeOH}$

b)

dMBzIm·EtOH
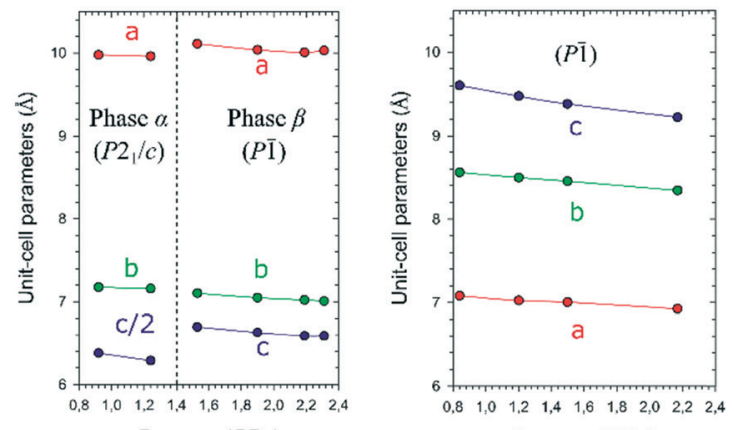

Pressure (GPa)
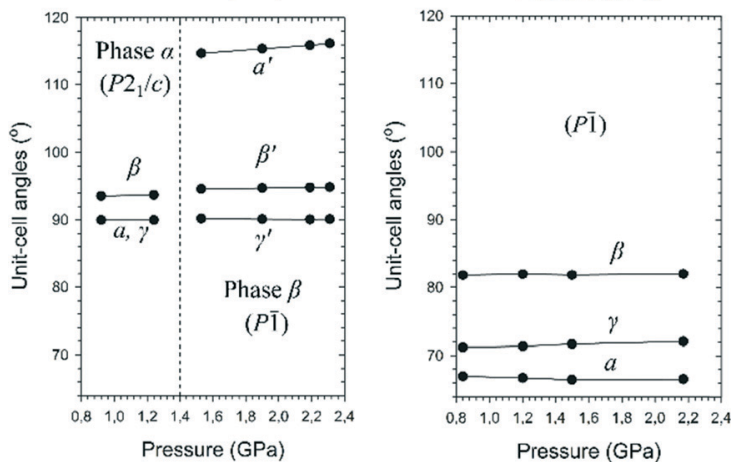

Fig. 2 Unit-cell parameters as a function of pressure in (a) dMBzlm.MeOH (the dashed line marks the transition between monoclinic and triclinic phases); and (b) dMBzIm.EtOH.

Another structural transformation in dMBzIm.MeOH at $P_{\mathrm{c}}$ is that in phase $\alpha$, the azole and hydroxyl H-atoms are disordered in the hydrogen bonds, while in phase $\beta$ they are ordered. This ordering of $\mathrm{H}$-atom results in the changed orientation of the dMBzIm and methanol molecules, in connection to the directional character of the hydrogen bonds, different at their $\mathrm{H}$-donor and $\mathrm{H}$-acceptor sites. ${ }^{16,17}$ The effect of the molecular orientation for the $\mathrm{H}$-sites in hydrogen bonds $\mathrm{NH}^{\cdots} \mathrm{OH} \cdots \mathrm{N}$ can be correlated to angles $\mathrm{C}-\mathrm{N} \cdots \mathrm{O}$ and $\mathrm{C}-\mathrm{O} \cdots \mathrm{N}$, also known as Donohue angles. ${ }^{18}$ Their a) $\alpha$-dMBzIm $\cdot \mathrm{MeOH}$

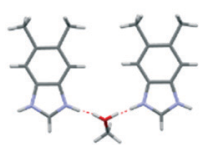

Space group $P 2_{1} / c$

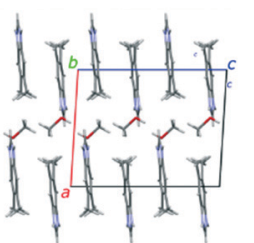

b)

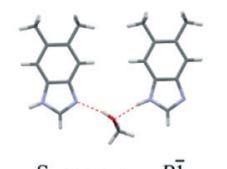

Space group $P \overline{1}$

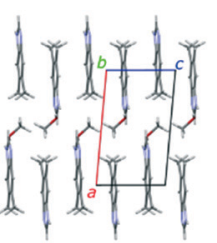

c) dMBzIm $\cdot \mathrm{EtOH}$

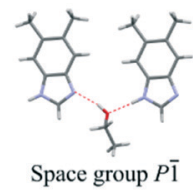

Space group $P \overline{1}$

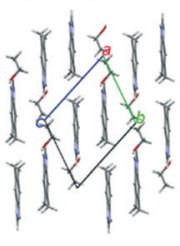

Fig. 3 Chains of $\mathrm{NH} \cdots \mathrm{OH} \cdots \mathrm{N}$ hydrogen bonded molecules in (a) dMBzIm.MeOH phase $\alpha$; (b) dMBzIm.MeOH phase $\beta$; and (c) $\mathrm{dMBzIm} \cdot \mathrm{EtOH}$. Also, the structures projected down the $\mathrm{NH} \cdots \mathrm{OH} \cdots \mathrm{N}$ bonded chains are shown in the bottom (the voids in these structures are shown in Fig. S1 in ESI $\dagger$ ). 

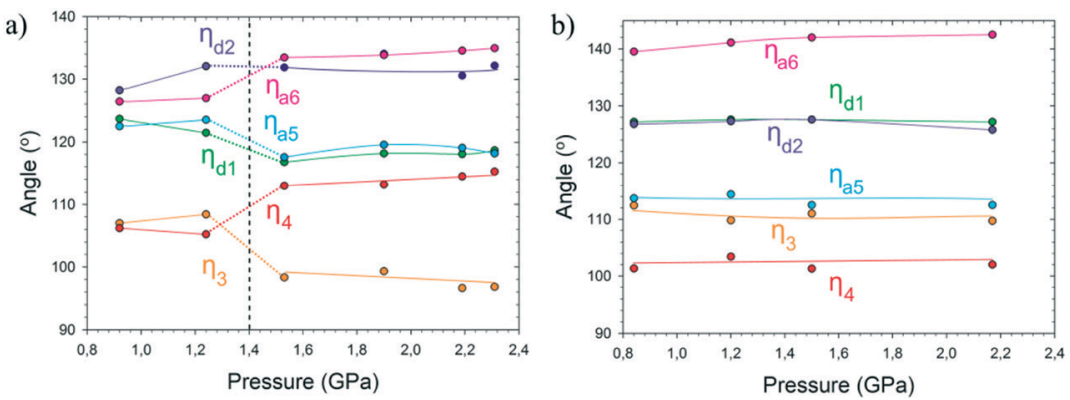

c)

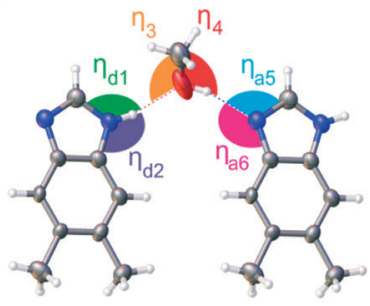

Fig. 4 Angles $\mathrm{C}-\mathrm{N} \cdots \mathrm{O}$ and $\mathrm{C}-\mathrm{O} \cdots \mathrm{N}$ in the $\mathrm{NH} \cdots \mathrm{OH} \cdots \mathrm{N}$ bonds in the crystal structures of the solvates: (a) dMBzlm.MeOH phases; (b) $\mathrm{dMBzIm} \cdot \mathrm{EtOH}$ as a function of pressure; and (c) the $\mathrm{NH} \cdots \mathrm{OH} \cdots \mathrm{N}$ bonds with indicated Donohue angles.

pressure dependence is shown in Fig. 4. It can be observed that the phase transition in dMBzIm·MeOH makes the angles on both sites of the methanol molecule significantly different, and one of these angles becomes much closer to the ideal angle of $109^{\circ}$, consistent with the hydroxyl H-atom position.

The origin of the preference between unsolvated and solvated crystallization of dMBzIm at different pressures can be connected with the interplay of transformed intermolecular interactions (H-bonds) as well as with the volume difference between the neat and solvated components. These volume changes are illustrated in Fig. 5. The volume difference between the solvate formula unit and the volume of one solvate-molecule assumed in the pure solvent under the same conditions for both the methanol solvate phases $\alpha$ and $\beta$ as well as for the ethanol solvate is smaller than that of the neat dMBzIm crystal. It means that the work-energy contribution $P \Delta V$ performed via pressure for the formation of the solvate is negative, equal to $-0.6 \mathrm{~kJ} \mathrm{~mol}^{-1}$, i.e. favorable for the solvate formation.

On the other hand, the volume increase $\Delta V$ of the solvates compared to that of neat dMBzIm is about $45 \AA^{3}$ for dMBzIm.MeOH and $70 \AA$ for dMBzIm.EtOH, which is consis-

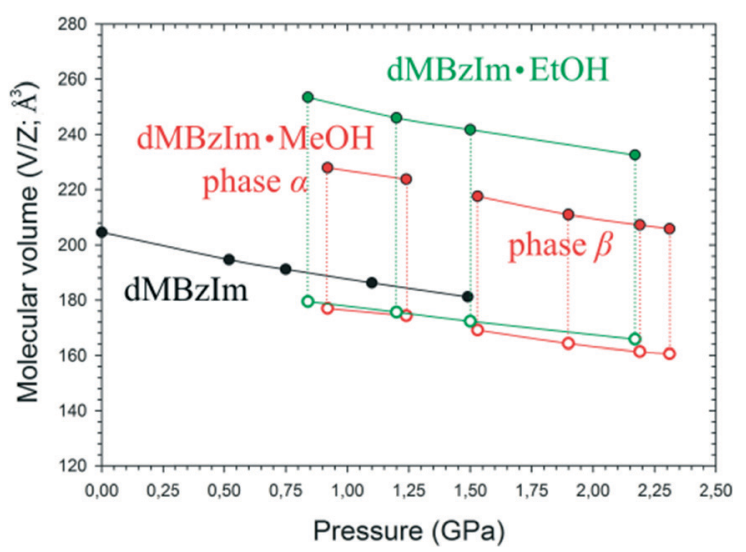

Fig. 5 Pressure dependence of the molecular volume (V/Z) of dMBzIm as well as its methanol $(\mathrm{MeOH})$ and ethanol $(\mathrm{EtOH})$ solvates. At 1.40 GPa dMBzIm.MeOH transforms from monoclinic space group $P 2_{1} / c$ to triclinic phase $\beta$ space group $P \overline{1}$. Dashed lines mark the molecular volume of $\mathrm{MeOH}$ and $\mathrm{EtOH}$ in their neat liquid form ${ }^{19}$ subtracted from the volume of the solvates. tent with such a volume increase in the solvates deposited in the Cambridge Structural Database. ${ }^{20}$ It is an indication that dMBzIm.MeOH and dMBzIm.EtOH do not differ significantly from other solvates obtained under normal conditions, although a high pressure is indispensable for their generation. However, according to our observations both of these solvates decompose below 0.60 GPa.
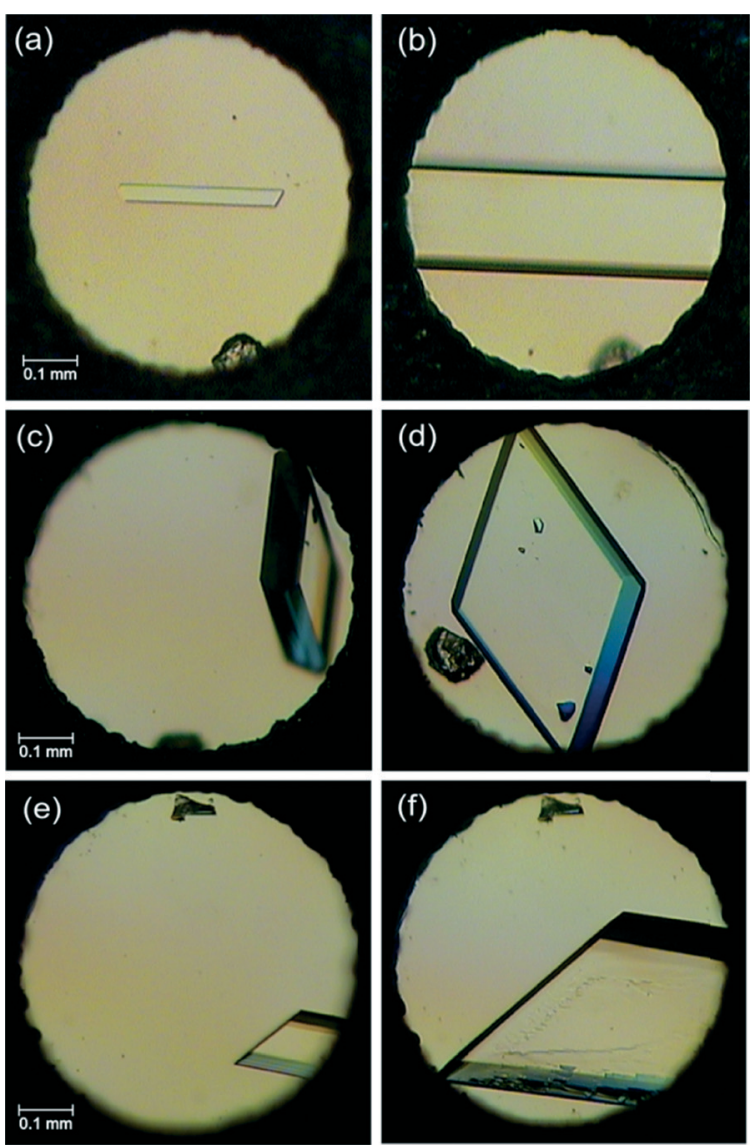

Fig. 6 Single crystals grown in situ inside the DAC chamber: (a-b) a neat dMBzIm crystal grown from the methanol solution at $0.22 \mathrm{GPa}$; (c-d) from the same solution, a dMBzlm.MeOH crystal at $0.92 \mathrm{GPa}$; and (e-f) from the ethanol solution, a dMBzlm.EtOH crystal grown at 1.42 GPa. Small irregular ruby chips in the chamber are for pressure calibration. The sample crystal and the ruby chips were loose in the chamber (c) before the crystal was jammed between the gasket edges (d). 


\section{Conclusions}

High pressure has been successfully used for inducing the solvation of dMBzIm with methanol and ethanol. In several respects it proceeds differently than it was previously observed for $\mathrm{dMBzIm} \cdot 1 / 2 \mathrm{H}_{2} \mathrm{O}$ hemihydrate. The analogy of the methanol and ethanol solvates with the hemihydrate includes also the substitution of $\mathrm{NH} \cdots \mathrm{N}$ bonds in neat dMBzIm with bonds $\mathrm{NH} \cdots \mathrm{OH} \cdots \mathrm{N}$ in the solvates. However the apparent difference between the hemihydrate and the solvates investigated is the magnitude of $\Delta V_{\mathrm{s}}$, much smaller for dMBzIm $\cdot 1 / 2 \mathrm{H}_{2} \mathrm{O}$, which agrees well with the stability of this hemihydrate under normal conditions. In this respect the pressure-induced solvation of dMBzIm considerably differs depending on the solvate, although the intermolecular interactions involved in the solvation are similar. Most importantly, it has been shown that apart from the $\mathrm{dMBzIm} \cdot 1 / 2 \mathrm{H}_{2} \mathrm{O}$ hemihydrate, the pressure provides an efficient method, and is the only one known so far for obtaining other solvates of 5,6-dimethylbenzimidazole.

\section{Experimental section}

\section{Crystal growth at high pressure}

5,6-Dimethylbenzimidazole (m.p. 475 K), analytical grade, from Acros Organics, was used as delivered. High pressure studies were performed in a Merrill-Bassett diamond-anvil cell (DAC) ${ }^{21}$ modified by mounting the diamond anvils directly on steel supports with conical windows; ${ }^{22}$ tungsten gaskets of a $0.2 \mathrm{~mm}$ thickness with a spark-eroded hole of 0.40 $\mathrm{mm}$ in diameter were used. Single crystals of dMBzIm.MeOH and dMBzIm·EtOH solvates were obtained above $0.6 \mathrm{GPa}$ from saturated solutions in methanol and ethanol, respectively. The pressure was calibrated via the ruby-fluorescence method $^{23}$ with a Photon Control Inc. spectrometer, affording an accuracy of $0.02 \mathrm{GPa}$, before and after the diffraction measurements. Single crystals were grown in isothermal and isochoric conditions in the DAC. A hot-air gun with a digital control of temperature, and an independent measurement of temperature using a thermocouple at one of the anvils inside the DAC were applied. The polycrystalline mass, obtained via isothermal compression, was heated until a single grain was left and then the sample was slowly cooled to room temperature. Single crystals of the dMBzIm solvates obtained in situ in the DAC are shown in Fig. 6.

\section{X-ray diffraction analyses}

The single-crystal diffraction data for dMBzIm at high pressures were measured at $296 \mathrm{~K}$ on a KUMA KM-4 CCD diffractometer with the graphite-monochromated MoK $\alpha$ radiation. The DAC was centered via the gasket-shadow method. ${ }^{24}$ The reflections were collected in the $\omega$-scan mode, $0.75^{\circ}$ frames and $30 \mathrm{~s}$ exposures. The CrysAlisCCD and CrysAlisRED programs $^{25}$ were used for data collection, determination of the UB-matrix, and for initial data reduction and Lp corrections; reflections intensities have been accounted for the effects of absorption of X-rays through the DAC, shadowing of the beams by the gasket edges, and absorption of the sample crystal itself using the program REDSHABS. ${ }^{26}$ The structures of the solvates were solved using direct methods. ${ }^{27}$ The OLEX2 program $^{28}$ with implemented SHELXL ${ }^{27}$ was used for all high-pressure data refinements. The positions of carbon $\mathrm{H}$-atoms were calculated from the molecular geometry assuming a C-H bond length of $0.93 \AA$ A.

The azole and hydroxyl hydrogen atoms were located from difference Fourier maps and then restrained to the molecular geometry by instructions AFIX 43 and AFIX 134 with the N-H distance of $0.86 \AA$ and $0.82 \AA$, respectively. Their location was also checked in this way, that the $\mathrm{H}$-atoms were constrained at the idealized positions at both $\mathrm{N}$-atoms, and then their site occupation factors (SOF) were refined with boundary condition assuming one $\mathrm{H}$-atom in each $\mathrm{H}$-bond, SOF(H1) + $\operatorname{SOF}(\mathrm{H} 3)=1$ for $\mathrm{N} 1-\mathrm{H} 1 \cdots \mathrm{N} 3{ }^{\prime}$ bond $(\mathrm{H} 3$ atom is the disordered $\mathrm{H}$-site at $\mathrm{N} 3$ atom); $\mathrm{SOF}(\mathrm{H} 1)+\mathrm{SOF}(\mathrm{H} 1 \mathrm{a})=1$ and $\mathrm{SOF}(\mathrm{H} 1 \mathrm{~b})+\mathrm{SOF}(\mathrm{H} 3)=1$ for $\mathrm{N} 1-\mathrm{H} 1 \cdots \mathrm{O} 1-\mathrm{H} 1 \mathrm{~b} \cdots \mathrm{N} 3^{\prime}$ (H1a and $\mathrm{H} 1 \mathrm{~b}$ are the sites of disordered hydroxyl $\mathrm{H}$-atoms in methanol and ethanol molecules). All these tests corroborated the ordered $\mathrm{H}$-atom position in the $\mathrm{NH} \cdots \mathrm{OH} \cdots \mathrm{N}$ bonds found in the difference Fourier maps: disordered azole and hydroxyl $\mathrm{H}$-locations in the low-pressure phase $\alpha$ of dMBzIm.MeOH are ordered in the $\beta$-phase and in dMBzIm.EtOH. In all plots and discussion of hydrogen bonding $\mathrm{NH} \cdots \mathrm{N}$ geometry the $\mathrm{H}$-positions were normalized to the $\mathrm{N}-\mathrm{H}$ bond length of $1.013 \AA, \mathrm{C}-\mathrm{H}$ of $1.099 \AA$ and $\mathrm{O}-\mathrm{H}$ of $0.98 \AA$ determined in neutron-diffraction studies. ${ }^{29}$ The crystallographic information of selected experiments is summarized in Table 1 (cf. Table $\mathrm{S} 1$ in ESI $\dagger$ ). The crystallographic data of the $\mathrm{dMBzIm} \cdot \mathrm{EtOH}$ structures at $0.84,1.20,1.50,2.17 \mathrm{GPa}$ and $\mathrm{dMBzIm} \cdot \mathrm{MeOH}$ structures at $0.92,2.24,1.53,1.90,2.19$, 2.31 GPa have been deposited to the Cambridge Crystallographic Database Center as supplementary publications CCDC 1451562-1451571, respectively. The molecular and intermolecular dimensions were calculated with programs OLEX2 (ref. 27) and Mercury. ${ }^{30}$

\section{Notes and references}

1 F. P. A. Fabbiani, D. R. Allan, W. I. David, S. A. Moggach, S. Parsons and C. R. Pulham, CrystEngComm, 2004, 6, 504.

2 P. Vishweshwar, J. A. McMahon, J. A. Bis and M. J. Zaworotko, J. Pharm. Sci., 2006, 95, 499.

3 E. Boldyreva, Z. Kristallogr., 2014, 229, 236.

4 F. P. A. Fabbiani, D. R. Allan, S. Parsons and C. R. Pulham, CrystEngComm, 2005, 7, 179.

5 H. Hirai, T. Kondo, M. Hasegawa, T. Yagi, Y. Yamamoto, T. Komai, K. Nagashima, M. Sakashita, H. Fujihisa and K. Aoki, J. Phys. Chem. B, 2000, 104, 1429.

6 B. P. van Eijck and J. Kroon, Acta Crystallogr., Sect. B: Struct. Sci., 2000, 56, 535.

7 H. H. Tung, E. L. Paul, M. Midler and J. A. McCauley, Crystallization of Organic Compounds: An Industrial Perspective, John Wiley \& Sons, Inc., Hoboken, New Jersey, 2009.

8 E. H. Lee, Asian J. Pharm. Sci., 2014, 9, 163. 
9 N. Shan and M. J. Zaworotko, Drug Discovery Today, 2008, 13, 440.

10 S. Dharmendra and C. William, Adv. Drug Delivery Rev., 2004, 56, 335.

11 G. R. Desiraju, J. Am. Chem. Soc., 2013, 135, 9952.

12 M. Anioła, A. Olejniczak and A. Katrusiak, Cryst. Growth Des., 2014, 14, 2187.

13 W. Zielinski and A. Katrusiak, Cryst. Growth Des., 2013, 13, 696.

14 W. Zielinski and A. Katrusiak, Cryst. Growth Des., 2014, 14(9), 4247.

15 W. Zielinski and A. Katrusiak, CrystEngComm, 2015, 17, 5468.

16 A. Katrusiak, M. Ratajczak-Sitarz and E. Grech, J. Mol. Struct., 1999, 474, 135.

17 M. Ratajczak-Sitarz, M. D. Rozwadowska and A. Katrusiak, Pol. J. Chem., 1998, 72, 2493.

$18 \mathrm{~J}$. Donohue, Selected topics in hydrogen bonding, in Structural Chemistry and Molecular Biology, ed. A. Rich and N. Davison, Freeman, San Francisco, 1968, pp. 443-465.

19 P. W. Bridgman, Proc. Am. Acad. Arts Sci., 1942, 74, 399.

20 M. Zaworotko, Am. Pharm. Outsorc., 2004, 5, 16.
21 L. Merrill and W. A. Bassett, Rev. Sci. Instrum., 1974, 45, 290.

22 A. Katrusiak, Acta Crystallogr., Sect. A: Found. Crystallogr., 2008, 64, 135.

23 H. K. Mao, J. Xu and P. M. Bell, J. Geophys. Res., B, 1986, 91, 4673.

24 A. Budzianowski and A. Katrusiak, High-Pressure Crystallographic Experiments with a CCD Detector, HighPressure Crystallography, ed. Katrusiak, A. and McMillan, P., Kluwer, Dordrecht, 2004.

25 CrysAlisCCD, CrysAlisRed, Oxford Diffraction, Oxford Diffraction Ltd, Abingdon, England, 2006.

26 A. Katrusiak, Z. Kristallogr., 2004, 219, 461.

27 G. M. Sheldrick, Acta Crystallogr., Sect. A: Found. Crystallogr., 2008, 64, 112.

28 O. V. Dolomanov, L. J. Bourhis, R. J. Gildea, J. A. K. Howard and H. Puschmann, J. Appl. Crystallogr., 2009, 42, 339.

29 F. H. Allen and I. J. Bruno, Acta Crystallogr., Sect. B: Struct. Sci., 2010, 66, 380.

30 C. F. Macrae, P. R. Edgington, P. McCabe, E. Pidcock, G. P. Shields, R. Taylor, M. Towler and J. van de Streek, J. Appl. Crystallogr., 2006, 39, 453. 